\title{
Epidemiologie des metabolischen Syndroms und des Typ-2-Diabetes \\ Primärprävention im Kindesalter notwendig
}

\author{
Dietrich Rothenbacher \\ Institut für Epidemiologie und Medizinische Biometrie, Universität Ulm, Ulm
}

Das metabolische Syndrom ist ein wichtiger Risikofaktor für die koronare Herzerkrankung und den Typ-2-Diabetes. Es setzt sich aus den Einzelsymptomen zentralbetonte Fettleibigkeit, Bluthochdruck, Dyslipidämie und Insulinresistenz zusammen. In Deutschland ist jeder Fünfte davon betroffen. Der Diabetes mellitus ist mit die häufigste Stoffwechselerkrankung in den industrialisierten Ländern. Auch durch den demografischen Wandel mit bedingt, nimmt die Häufigkeit der Erkrankung weltweit zu. In Deutschland leiden etwa $5 \%$ der Bevölkerung an einem Diabetes, $90 \%$ davon haben den Typ-2-Diabetes. Die Häufigkeit steigt ab dem 50. Lebensjahr steil an. Mindestens 2 Drittel der Typ-2-Diabetiker haben zusätzlich zu ihrer Grunderkrankung noch andere Krankheiten wie Hypertonie und Fettstoffwechselstörungen, die dringend einer Behandlung bedürfen. Personen mit einem metabolischen Syndrom und/oder Diabetes haben ein besonders hohes Risiko für arteriosklerotisch bedingte Folgekrankheiten, ca. 75-80\% der Diabetiker sterben an kardiovaskulären Herzerkrankungen. Neben genetischen Risikofaktoren und dem Alter spielen modifizierbare Risikofaktoren eine ganz entscheidende Rolle in der Kausalität der Diabetes-assoziierten Morbidität und Mortalität. Speziell eine Erhöhung der körperlichen Aktivität, eine gesunde Ernährung und eine Gewichtsreduktion sind aussichtsreiche Präventionsziele. Diese sollten aber bereits sehr früh im Kindesalter einsetzen und in die Lebenswelten aller gesellschaftlichen Gruppen integriert werden.

Das metabolische Syndrom ist ein wichtiger Risikofaktor für die koronare Herzerkrankung und den Typ-2-Diabetes. Es setzt sich aus den Einzelsymptomen zentralbetonte Fettleibigkeit, Bluthochdruck, Dyslipidämie, und Insulinresistenz zusammen [1]. Beim gleichzeitigen Vorhandensein dieser Faktoren steigt das Risiko deutlich an. Derzeit wird allerdings diskutiert, ob dieses Syndrom das Risiko von kardiovaskulären Ereignissen besser vorhersagt, als die Summe der Einzelkomponenten [2]. Lebensstilfaktoren wie Mangel an körperlicher Aktivität und hyperkalorische Ernährung sind hauptsächliche Einflussgrößen und machen das metabolische Syndrom zu einem speziellen Problem in den industrialisierten und den sogenannten Schwellenländern. In letzteren kommt es vor allem durch eine Verwestlichung des Lebensstils mit einer hochkalorischen Ernährung und verminderter körperlichen Aktivität zu einer regelrechten Epidemie in den nächsten Jahren [3].

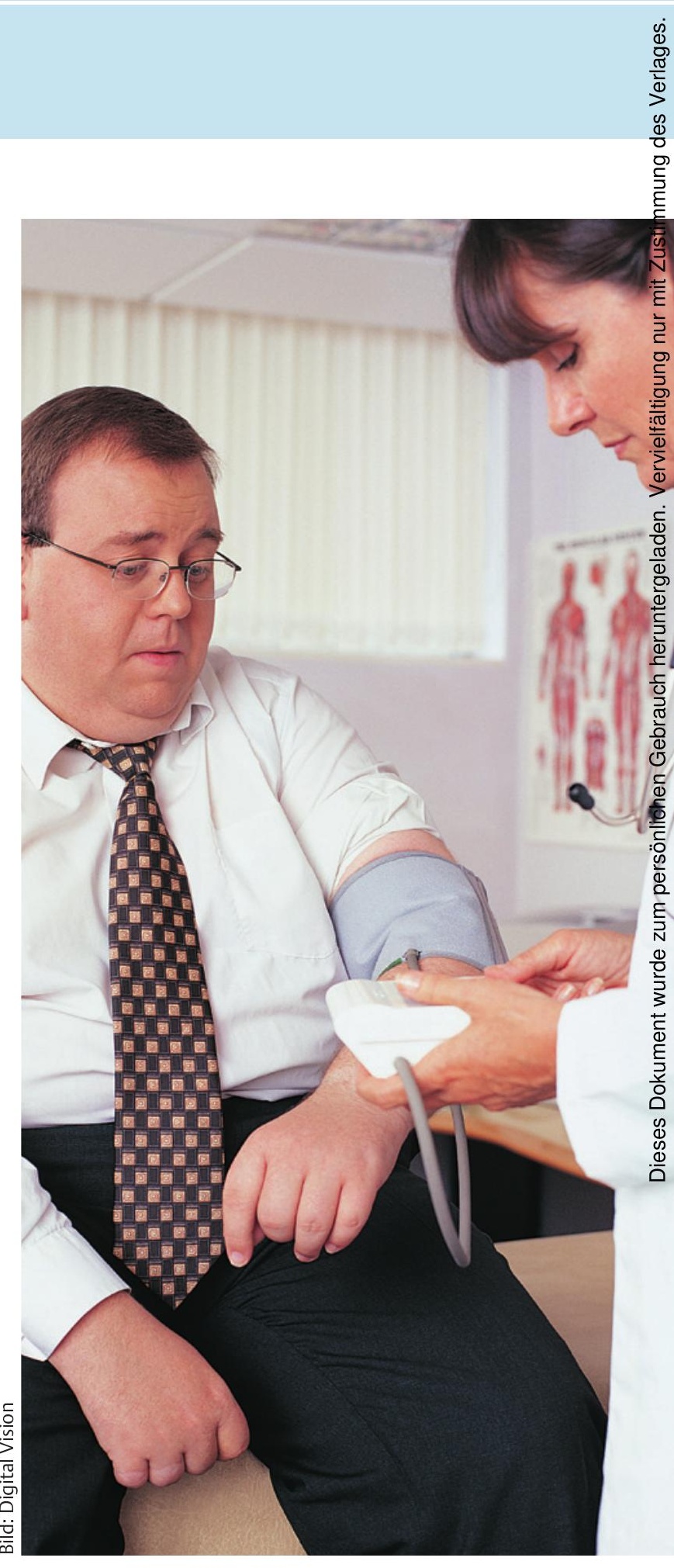


Eine allgemein anerkannte Definition des metabolischen Syndroms gibt es bisher nicht. Nach der ursprünglichen Definition der Weltgesundheitsorganisation (WHO) liegt ein metabolisches Syndrom dann vor, wenn alle 3 nachfolgenden Risikofaktoren vorhanden sind: Diabetes mellitus, ein gestörte Glukosetoleranz, ein pathologischer Nüchternblutzucker, bzw. eine Insulinresistenz [1]. Zudem sind 2 der 3 folgenden Befunde notwendig: arterielle Hypertonie, eine Dyslipidämie, oder eine viszerale Adipositas.

Durch weitere Erkenntnisse der relevanten $\mathrm{Zu}$ sammenhänge sind weitere Definitionen hinzugekommen. Die Tabelle 1 zeigt die Details gängiger Definitionen. Mittlerweile ist erkannt worden, dass es nicht das Übergewicht als solches, sondern vor allem die Fettverteilung ist, die das nachfolgende Risiko von kardiovaskulären Erkrankungen stark beeinflusst. So ist es vor allem das viszerale Fett, das spezielle endokrine Funktionen zu haben scheint, und sich hier über die Einbeziehung des Taillenumfangs besser als Risikoprädiktor einbeziehen lässt.

Die Definition des metabolischen Syndroms der Internationalen Diabetes Foundation (IDF) wird allgemein akzeptiert und immer öfter in internationalen Untersuchungen verwendet [1]. In diese Definition gehen Grenzwerte für den Bauchumfang als obligatorische Parameter mit ein. Außerhalb von speziellen Studien ist es schwer, Daten zur Epidemiologie des metabolischen Syndroms zu erhalten, da kein allgemein akzeptierter Code zur Verschlüsselung existiert, sondern nur Informationen über die einzelnen Manifestationen erhältlich sind.

\section{Epidemiologie des metabolischen Syndroms}

In Deutschland erfüllt circa jeder 5. Bundesbürger die Kriterien des metabolischen Syndroms wie aus den Daten des nationalen Gesundheitssurvey aus dem Jahr 1998 deutlich wurde [5]. Dabei wurden die Kriterien des National Cholesterol Education Program (NCE) angewendet, wie diese im „Third Report of the Expert Panel on Detection, Evaluation, and Treatment of High Blood Cholesterol in Adults“ (NCEP-ATP-III) formuliert wurden [1]. Eine neuere Untersuchung an 35869 Patienten im Alter von über 18 Jahren aus primärärztlichen Praxen Deutschlands kommt zu ähnlichen Ergebnissen [6]. Die Prävalenz bei den Frauen betrug dabei $18,0 \%$, bei den Männern $22,7 \%$. In der Studie wurden auch ein deutlicher, positiver Gradient mit dem Alter und eine inverse Beziehung mit der Bildung beschrieben. Es zeigten sich starke regionale Zusammenhänge, so waren höhere Zahlen in den östlichen Bundesländern zu finden. In Baden-Württemberg waren insgesamt 17,1 \% der Frauen und 21,1 \% der Männer vom metabolischen Syndrom betroffen.

\section{Tab. 1 Entwicklung der Begriffsdefinitionen des metabolischen Syndroms [nach 1].}

\begin{tabular}{|c|c|}
\hline Wer, wann & Kriterien \\
\hline \multirow{3}{*}{$\begin{array}{l}\text { Weltgesundheitsorganisation } \\
\text { (WHO), } 1989\end{array}$} & Diabetes mellitus, \\
\hline & eine gestörte Glukosetoleranz, \\
\hline & 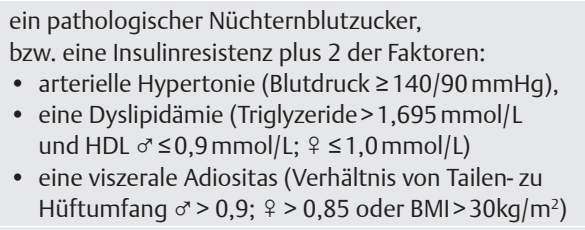 \\
\hline \multirow{4}{*}{$\begin{array}{l}\text { National Cholesterol Education } \\
\text { Program (NCE) Third Report of } \\
\text { the Expert Panel on Detection, } \\
\text { Evaluation, and Treatment of High } \\
\text { Blood Cholesterol in Adults (ATP-III) } \\
\text { NCEP-ATP-III, } 2001\end{array}$} & $\begin{array}{l}\text { abdominelle Fettverteilung } \\
\text { (Bauchumfang ơ } \geq 102 \mathrm{~cm} ; \text { ㅇ } \geq 88 \mathrm{~cm} \text { ) }\end{array}$ \\
\hline & Triglyceride (>150 mg/dL) \\
\hline & $\mathrm{HDL}\left(\sigma^{\pi}<40 \mathrm{mg} / \mathrm{dL} ; \circ<50 \mathrm{mg} / \mathrm{dL}\right)$ \\
\hline & arterielle Hypertonie (Blutdruck $\geq 130 / 85 \mathrm{mmHg}$ ) \\
\hline \multirow[t]{2}{*}{$\begin{array}{l}\text { International Diabetes Foundation } \\
\text { (IDF), } 2005\end{array}$} & $\begin{array}{l}\text { bauchbetonte, zentrale Fettleibigkeit } \\
\text { ( } \sigma^{\star} \geq 94 \mathrm{~cm} ; \text { \& } \geq 80 \mathrm{~cm} \text { Taillenumfang) }\end{array}$ \\
\hline & $\begin{array}{l}\text { plus } 2 \text { Faktoren: } \\
\text { - Nüchternblutzucker }>100 \mathrm{mg} / \mathrm{dl} \text { oder Diabetes } \\
\text { - Triglyceride }>150 \mathrm{mg} / \mathrm{dL} \text { (oder Medikation) } \\
\text { - } \mathrm{HDL} \mathrm{o}^{\pi}<40 \mathrm{mg} / \mathrm{dl}, \text { o }<50 \mathrm{mg} / \mathrm{dL} \text { (oder Medikation) } \\
\text { - Bluthochdruck (ab } 130 \mathrm{mmHg} \text { syst. und }>85 \mathrm{mmHg} \\
\text { diastolisch) (oder Therapie) }\end{array}$ \\
\hline
\end{tabular}

Schwieriger ist es Daten von Kindern zu erhalten. Hier kann aber ein Blick auf die Einzelkomponenten des metabolischen Syndroms helfen, aktuelle und zukünftige Entwicklungen der Krankheitslast abzuschätzen. Die Daten der bevölkerungsweit durchgeführten Einschulungsuntersuchungen sind dabei ein sehr wertvolles Instrument, das es erlaubt, alle Kinder eines Jahrgangs in geografisch definierten Regionen zu erfassen. Dabei kann quasi von einer Vollerhebung ausgegangen werden.

Eine Auswertung der verfügbaren Daten der einzelnen Bundesländer von Moss und Kollegen zeigt bereits im Vorschulalter starke regionale Unterschiede der Prävalenz des Übergewichts, bzw. der Adipositas [7]. So wurde die höchste Häufigkeit von 11,6 bzw. 5,4\% im Saarland beschrieben, in Baden-Württemberg lag sie bei 9,6\%, bzw. 3,7\%. Am niedrigsten war die Häufigkeit des Übergewichts in Sachsen und Brandenburg mit jeweils 8,4\% und 8,5\% ausgefallen. Eine Adipositas fand sich in beiden Ländern bei 3,3\%. Daten aus dem Kinder- und Jugendsurvey zeigen, dass 15\% der Kinder und Jugendlichen im Alter von 3 bis 17 Jahren übergewichtig sind [8]. Die Daten zeigen einen besonders starken Anstieg des Übergewichts bei den 7-10-jährigen Kindern im Vergleich zu den Vorschulkindern. Hier wird deutlich, dass vor allem Primärpräventionsstrategien bereits im frühen Kindesalter ansetzen sollten, um zu wirken und weisen damit das Vorschulkinderalter oder die ersten Schuljahre als spezielles „window of opportunity“ aus. Immer mehr Förderprogramme zielen deshalb auf Kindertagesstätten und Kindergärten $a b$, um gesunde Ernährung und ausreichende Bewegung als Erziehungs- und Gesund- 


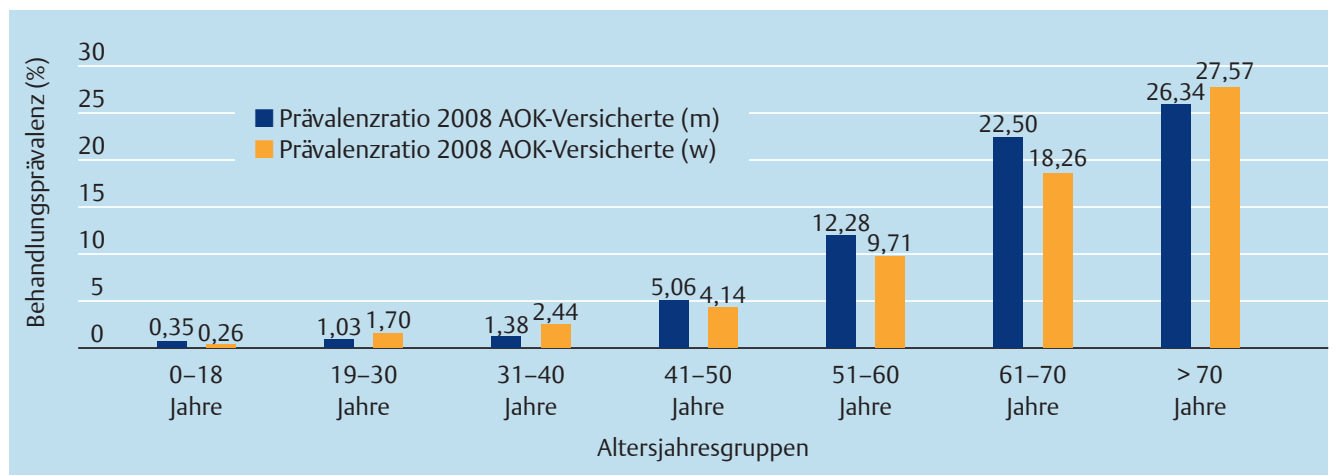

Abb. 1 Prävalenz des Typ-2-Diabetes bei 61-70-jährigen Versicherten der AOK [nach 10].

heitsförderkonzepte in den Alltag von Kindern zu integrieren.

Deutlich wird in allen Untersuchungen auch eine besondere Betroffenheit der Kinder mit Migrationshintergrund. Die gute Nachricht der Studie von Moss und Kollegen [7] ist, dass die Prävalenz des Übergewichts und der Adipositas in den letzten 4-6 Jahren nicht weiter angestiegen ist, sondern teilweise sogar leicht rückläufig scheint. Ein Vergleich der Daten aus dem KIGGS mit Referenzwerten aus den 1980er und 1990ern zeigte zuvor einen 50\%igen Anstieg.

\section{Epidemiologie des Diabetes mellitus}

Der Diabetes mellitus ist mit die häufigste Stoffwechselerkrankung in den industrialisierten Ländern. Auch durch den demografischen Wandel mitbedingt, nimmt die Häufigkeit der Erkrankung weltweit zu [3]. In Deutschland leiden etwa 5\% der Bevölkerung an einem Diabetes, $90 \%$ davon haben den Typ-2-Diabetes. In einer Untersuchung in 12 Hausarztpraxen im Raum Ludwigsburg lag die Prävalenz des Diabetes mellitus im Patientengesamtkollektiv aller beteiligten Praxen bei 6,4\% [9]. Die Häufigkeit steigt ab

\begin{tabular}{|c|c|}
\hline $\begin{array}{l}\text { Bekannte Begleiterkrankungen } \\
\text { zum Diabetes }\end{array}$ & $\begin{array}{l}\text { Gesamt } \\
n=1065\end{array}$ \\
\hline Hypercholesterinämie & $53 \%$ \\
\hline arterielle Hypertonie & $71 \%$ \\
\hline koronare Herzerkrankung & $29 \%$ \\
\hline Herzinfarkt & $6 \%$ \\
\hline Schlaganfall & $11 \%$ \\
\hline $\begin{array}{l}\text { periphere arterielle } \\
\text { Verschlusskrankheit }\end{array}$ & $14 \%$ \\
\hline Mikroalbuminurie & $23 \%$ \\
\hline Retinopathie & $14 \%$ \\
\hline \multicolumn{2}{|l|}{ Sonstige Medikation } \\
\hline ACE-Hemmer & $45 \%$ \\
\hline Betablocker & $23 \%$ \\
\hline Diuretika & $41 \%$ \\
\hline Acetylsalicylsäure (ASS) & $23 \%$ \\
\hline Lipidsenker & $12 \%$ \\
\hline Sonstige Koronartherapeutika & $24 \%$ \\
\hline
\end{tabular}

dem 50. Lebensjahr steil an. Unter den männlichen 61-70-jährigen Versicherten der AOK Baden-Württemberg lag die Prävalenz bereits bei circa $22 \%$ und nimmt im höheren Lebensalter weiter zu (Abb. 1) [10].

Mindestens 2 Drittel der Typ-2-Diabetiker haben zusätzlich zu ihrer Grunderkrankung noch andere Krankheiten wie Hypertonie und Fettstoffwechselstörungen [11]. Personen mit einem metabolischen Syndrom und/oder Diabetes haben ein besonders hohes Risiko für arteriosklerotisch bedingte Folgekrankheiten. Circa 75-80\% der Diabetiker sterben an kardiovaskulären Erkrankungen [4]. Weitere Folgekrankheiten sind die Nephropathie mit der Gefahr der Dialysepflichtigkeit, die Retinopathie mit der Gefahr der Erblindung, die periphere arterielle Verschlusskrankheit und die Neuropathie, die bis hin zur Amputation der betroffenen Gliedmaßen führen kann.

\section{Ansatzpunkte für Prävention und Früherkennung}

Das Risiko des Diabetes ist modifizierbar. Neben genetischen Risikofaktoren und dem Alter spielen modifizierbare Risikofaktoren eine ganz entscheidende Rolle in der Kausalität der Diabetes-assoziierten Morbidität und Mortalität. Speziell eine Erhöhung der körperlichen Aktivität, eine gesunde Ernährung und eine Gewichtsreduktion sind aussichtsreiche Ziele einer Primärprävention [4]. Mittlerweile ist durch viele randomisierte Studien belegt, dass sowohl durch Änderung der Ernährungsgewohnheiten und durch mehr körperliche Aktivität als auch durch pharmakologische Konzepte wie beispielsweise die Gabe von Metformin eine Reduzierung des Diabetes zu erreichen ist; die erzielte Risikoreduktion fiel dabei mit den nicht-pharmakologischen Konzepten am höchsten aus [12].

Da mittlerweile ein Großteil unserer Bevölkerungen gefährdet ist, sind Präventionsmaßnahmen auf Bevölkerungsebene notwendig. Diese sollten die identifizierten Risikofaktoren betreffen und möglichst früh im Kindesalter ansetzen. 
Zudem sollten passende Programme auch für Jugendliche, Erwachsene und ältere Personen entwickelt werden und in deren Lebenswelten integriert werden. Diese Programme dürfen nicht nur auf den Einzelnen ausgerichtet sein, sondern müssen auch infrastrukturelle, kommunale und planerische Aspekte beinhalten, die unsere Schulen, Gebäude sowie öffentliche Lebensräume betreffen, um wieder eine Umgebung zu schaffen, die beispielsweise wieder das Spielen im Freien oder die Benutzung des Treppenhauses als selbstverständliche und angenehme Tätigkeit erfahrbar machen.

Zudem sollte in der Praxis auch eine konsequentere Behandlung von kardiovaskulären Risikofaktoren im Sinne der Sekundärprävention erfolgen. Wie frühere Untersuchungen gezeigt haben, ist die medikamentöse Betreuung der Patienten gemessen an den Empfehlungen der Fachgesellschaften noch nicht optimal (Tab. 2) [9, 13].

Eine konsequentere Behandlung von Hypertonie und Fettstoffwechselstörungen ist mit einer Reduzierung der Diabetes-assoziierten kardiovaskulären Erkrankungen, die bei $75-80 \%$ der Patienten zum Tode führen, verbunden. Dies ist auch bei älteren Patienten der Fall.

Aber auch neue Konzepte zur Früherkennung von Personen mit einem erhöhten Risiko können zu einer früheren Therapie und einer Senkung der assoziierten Morbidität genutzt werden [14]. So könnte die Einbeziehung des $\mathrm{HbA}_{1 \mathrm{c}}$ zum Nüchternglukosewert als Früherkennungsmaßnahme zur Identifizierung von Risikopersonen einen Mehrwert bedeuten.

Neben großem, persönlichem Leid resultieren aus diesen Krankheiten ein immenser Versorgungsbedarf und erhebliche direkte und indirekte Folgekosten für die Gesellschaft. Auch mit aus diesen Gründen ist eine verbesserte Prävention medizinisch und sozialpolitisch von größter Bedeutung. Ein Erfolg in diesen Bereichen scheint wahrscheinlicher, wenn es gelingt, die verschiedensten Disziplinen in diese Aufgabe einzubeziehen und die Lebenswelten unserer Gesellschaft so zu gestalten, dass Präventionsbedürfnisse sich als selbstverständliche Verrichtungen und Gegebenheiten in den Alltag integrieren lassen. Das kann uns nur gelingen, wenn wir vermitteln können, dass diese Präventionsaufgaben nicht nur als medizinische, sondern als fundamentale Aufgaben unserer ganzen Gesellschaft anzusehen sind, auch um die Zukunftsfähigkeit unserer Gesellschaft in einer globalisierten Welt langfristig sicher zu stellen.

\section{Literatur}

1 Kassi E, Pervanidou P, Kaltsas G, Chrousos G. Metabolic syndrome: definitions and controversies. BMC Medicine 2011; 9: 48

2 Eckel RH, Alberti KGMM, Grundy SM, Zimmet P. The metabolic syndrome. Lancet 2010; 375: 181-183
Epidemiology of metabolic syndrome and type 2 diabetes -

Prevention in childhood is necessary

The metabolic syndrome is an important risk factor for cardiovascular diseases and diabetes. It consists of the components central obesity, hypertension, dyslipidemia, and insulin resistance. In Germany every fifth person is affected. Diabetes mellitus is among the most common endocrine disorders in the industrialized countries. Also because of the demographic transition the prevalence will increase further on a world wide scale. About $5 \%$ of the population of Germany suffer from diabetes, $90 \%$ of them have a type 2 diabetes. The prevalence of diabetes increases steeply after the age of 50 years. About $2 / 3$ of patients with diabetes suffer from additional comorbidity such as hypertension or dyslipidemia, which require additional treatment. Subjects with metabolic syndrome and/or diabetes have an extreme risk of atherosclerotic diseases and about 75-80\% die because of cardiovascular heart diseases. Modifiable factors are playing a major role in causality of related morbidity and mortality beside genetic risk factors and age. An increase of physical activity, a healthy diet and reduction of overweight are promising targets for prevention. The latter should start, however, early in childhood and ideally be integrated in the daily life of all groups within society.

Key words

metabolic syndrome - diabetes - epidemiology - risk factors - prevention

3 Nolan C, Damm P, Prentki M. Type 2 diabetes across generations: from pathophysiology to prevention and management. Lancet 2011; 378: 169-181

4 Alberti KG, Zimmet P, Shaw J. The metabolic syndrome - a new worldwide definition. Lancet 2005; 366: 1059-1062

5 Neuhauser HK, Elert U. Prävalenz des metabolischen Syndroms in Deutschland. Eine Sensitivitätsanalyse. Gmds German Medical Science 2005. Meeting abstract von http://www.egms.de/static/en/meetings/ gmds2005/05gmds183.shtml

6 Moebus S, Hanisch J, Bramlage P et al. Regional unterschiedliche Prävalenz des metabolischen Syndroms. Daten zur primärärztlichen Versorgung in Deutschland. Dtsch Arztebl 2008; 105: A207-213

7 Moss A, Klenk J, Simon K et al. Declining prevalence rates for overweight and obesity in German children starting school. Eur J Peditr (Published online 13. Juli 2011, DOI 10.1007/s00431-011-1531-5)

8 Kurth BM, Schaffrath Rosario A. Übergewicht und Adipositas bei Kindern und Jugendlichen in Deutschland. Bundesgesundheitsbl 2010; 53: 643-652

9 Rothenbacher D, Rüter G, Saam S, Brenner H. Versorgung von Patienten mit Typ-2 Diabetes. Ergebnisse aus 12 Hausarztpraxen. Dtsch Med Wochenschr 2002; 127:1183-1187

10 Boehm B, Hoberg R, Hermann C et al. Prävalenz des Diabetes mellitus Typ 1 und Typ 2 in BadenWürttemberg: Eine Analyse der Versicherten der AOK Baden-Württemberg 2006-2008 (Abstrakt). Diabetologie \& Stoffwechsel 2010; 5: 184-185

11 Rothenbacher D, Brenner H, Rüter G. Typ-2-Diabetes-mellitus: Betreuung von chronisch Kranken in der Hausarztpraxis. Dtsch Arztebl 2005; 102: A240812

12 Iqbal N. The burden of type 2 diabetes: strategies to prevent or delay onset. Vasc Health Risk Management 2007; 3: 511-520

13 Raum E, Lietzau S, Stegmaier C et al. For the majority of patients with diabetes blood pressure and lipid management is not in line with recommendations. Results from a large population-based cohort in Germany. Pharmacoepi Drug Safety 2008; 17: 485-494

14 Schöttker B, Raum E, Rothenbacher D et al. Prognostic value of haemoglobin and fasting plasma glucose for incident diabetes and implications for screening: results of the ESTHER study. Eur J Epidemiol 2011; 26: 779-787

\section{Korrespondenz}

Prof. Dr. med. Dietrich

Rothenbacher, MPH

Institut für Epidemiologie und

Medizinische Biometrie,

Universität Ulm

Helmholtzstr. 22

89081 Ulm

dietrich.rothenbacher@uni-ulm. de

\section{Autorenerklärung}

Der Autor erklärt, dass für diesen Artikel keine Interessenkonflikte bestehen. 